\title{
Intervention by Caregiver
}

National Cancer Institute

\section{Source}

National Cancer Institute. Intervention by Caregiver. NCI Thesaurus. Code C116525.

An act of intercession or assistance for an individual that is performed by their caregiver. 